\title{
Transurethral endoscopic approach for large bladder diverticula: Evaluation of a large series
}

\author{
Mauro Pacella ${ }^{1}$, Nicolò Testino ${ }^{1}$, Guglielmo Mantica ${ }^{2}$, Matteo Valcalda ${ }^{1}$, Rafaela Malinaric ${ }^{1}$, \\ Carlo Terrone ${ }^{1}$ \\ ${ }^{1}$ Department of Urology, Policlinico San Martino Hospital, University of Genova, Genova, Italy; \\ ${ }^{2}$ Department of Urology, San Raffaele Turro Hospital, San Raffaele University, Milan, Italy.
}

\begin{abstract}
Summary Objective: To present the results of the largest series of patients with bladder diverticula $>4 \mathrm{~cm}$ managed with an endoscopic approach and give tips about the execution of the procedure.

Materials and methods: Data of male patients undergone the endoscopic approach for an acquired bladder diverticula $>4 \mathrm{~cm}$ from December 2004 to August 2018 were prospectively collected and retrospectively analyzed. The description of the monopolar and bipolar techniques are provided. The success of the procedure was defined as the reduction of the diverticula for more of the $80 \%$ of its initial diameter documented at the 3months follow-up imaging. Continuous variables with nonparametric distribution were compared using the Mann-Whitney test, while frequencies of categorical variables were compared between groups by Fisher's exact test with significance level set at 0.05 .

Results: Thirty-nine patients with a mean (+/- SD) age at surgery of $69.4 \pm 8.8$ years were enrolled, for an equal number of diverticula managed. The mean diverticular size was $75.1 \pm$ 24.5 millimeters. The mean operative time was $65 \pm 21.9$ minutes including the prostate surgery. Twelve patients (30.8\%) were managed with bipolar energy, the others with monopolar. The success of the procedure was achieved in 30 patients (76.9\% - 7 bipolar and 23 monopolar - $p=0.66$ ).

Conclusions: The endoscopic approach might be considered as a useful option for patients with a large bladder diverticulum who are at risk for major or laparoscopic procedures.
\end{abstract}

KEY WORDS: Bladder diverticula; BPH; Urinary retention; HoLEP; TURP.

Submitted 21 April 2019; Accepted 2 May 2019

\section{INTRODUCTION}

Acquired bladder diverticula are a herniation of the mucosa through the detrusor muscle and are mainly secondary to an outlet obstruction typically caused by benign prostate enlargement, bladder neck contracture and urethral stenosis. For these reasons, acquired bladder diverticula are usually a disease affecting the elderly male population. The surgical management of a bladder diverticulum itself is not always mandatory but often, especially if of big dimensions, they can lead to post void residual urine, lithiasis, urinary tract infections and ureteral compression. Mandatory should be the resolution of the obstruction, while the surgical management of the diverticula is mostly carried out through an open, laparoscopic or robotic approach. The endoscopic approach using the Orandi technique is usually reserved, during other endoscopic procedures, to diverticula less than $4 \mathrm{~cm}$ diameter, which are less likely to cause symptoms (1-3). Recently, some authors have proposed the endoscopic management with good success also for large diverticula (4-6).

We aim to present the results of the largest series of patients with bladder diverticula $>4 \mathrm{~cm}$ managed with an endoscopic approach and give tips about the execution of the procedure.

\section{Materials AND Methods}

The characteristics, intra- and peri- operative data, as well as the follow-up of male patients undergone the endoscopic approach for an acquired bladder diverticula from December 2004 to August 2018 were prospectively collected and retrospectively analyzed. The inclusion criteria were the presence of at least a diverticulum with a major diameter $>4 \mathrm{~cm}$ in a patient with comorbidities, an American Society of Anesthesiology Score (ASA Score) $\geq$ 2 and a Charlson Comorbidity index $\geq 3$. The preoperative assessment included routine blood tests with serum creatinine, urinalysis, Trans-rectal Ultrasound (TRUS), and CT scan or conventional cystogram, uroflometry, International Prostatic Symptoms Score (IPSS), serum Prostate Specific Antigen (PSA), serum creatinine, urinalysis and urine culture. At a 3 months follow-up all patients included in the study underwent a control cystogram or Kidney-Ureter-Bladder (KUB) ultrasound.

The success of the procedure was defined as the reduction of the diverticula for more of the $80 \%$ of its initial diameter. Complications were graded using the ClavienDindo classification (7).

Surgery is carried out with the patient in lithotomic position. A meticulous cystoscopy is performed and the subsequent step consist in the fulguration of the whole diverticular mucosa with a rollerball electrode using either the monopolar or bipolar energy source, with a coagulation setting respectively of 60-70 $\mathrm{W}$ and 70-80 W. The following step is the incision of the diverticular neck in four different cardinal points (12-3-6-9) and then the flattening of the entire diverticular neck circumference with the same rollerball electrode using plasma vaporization 
(160 W for monopolar, $180 \mathrm{~W}$ for bipolar). Subsequently the management of the primary obstruction is carried out (either with a TURP, TUIP, or other endoscopic procedures) and a 3 ways catheter inserted. All cases were performed by a single urologist (M.P.).

An antibiotic prophylaxis (cefazolin $1000 \mathrm{mg}$ ), or according to the urinalysis, was started 30 min before surgery. Antithrombotic prophylaxis with low-molecular weight heparin was administered when needed according to our Department protocol.

A 3 months follow up included conventional or CT cystography and abdominal, in addition to physical examination, uroflowmetry and IPSS.

Demographic and clinical characteristics and perioperative data were expressed as the mean \pm standard deviation (SD), median with interquantile range (IQR) or as count with percentage. Continuous variables with nonparametric distribution were compared using the Mann-Whitney test, while frequencies of categorical variables were compared between groups by Fisher's exact test with significance level set at 0.05. Data were analyzed with Sofastat TM for windows.

\section{RESULTS}

Thirty-nine patients with a mean (+/- SD) age at surgery of $69.4 \pm 8.8$ years were enrolled, for an equal number of diverticula managed. The mean diverticular size was $75.1 \pm 24.5$ millimeters. Six patients (15.4\%) underwent general anesthesia while $33(84.6 \%)$ spinal anesthesia. Eight patients (20.5\%) had a TUIP while $31(79.5 \%)$ a TURP. The mean operative time was $65 \pm 21.9$ minutes including the prostate surgery.

Twelve patients $(30.8 \%)$ were managed with bipolar energy, the others with monopolar. The success of the

Figure 1.

Patient A - Progressive reduction of the diverticular diameter. Images at 0-6-12 weeks.
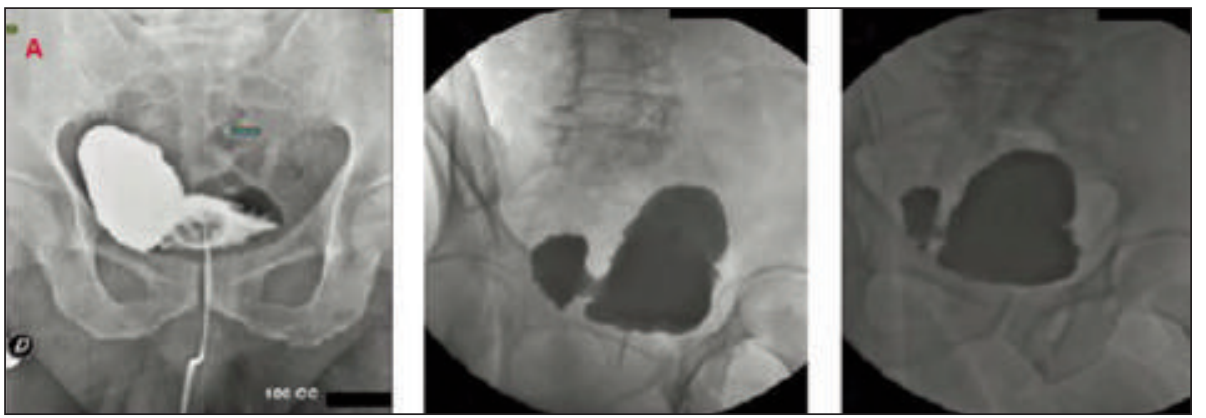

Figure 2.

Patient B - Complete disappearance of a bladder diverticulum at a CT-Scan performed 3 years later for other reasons.
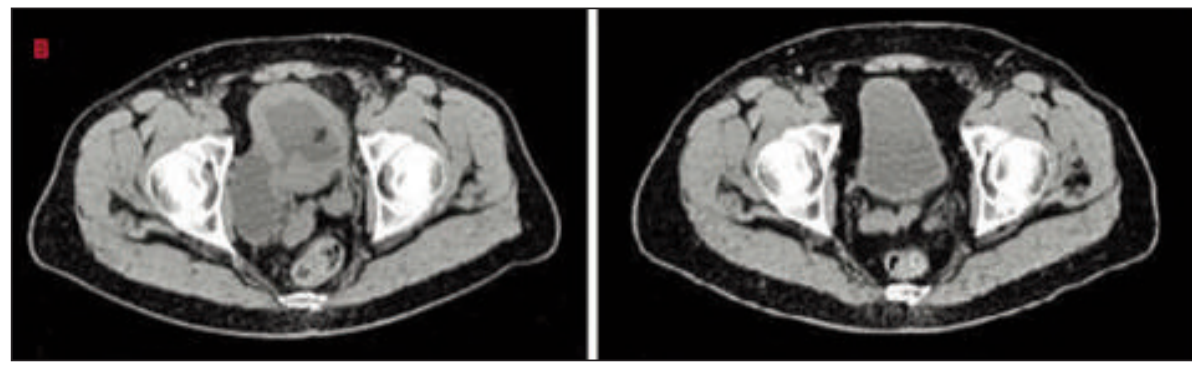

procedure was achieved in 30 patients ( $76.9 \%$ - 7 bipolar and 23 monopolar - $\mathrm{p}=0.66$ - Groups not significantly different in terms of age, ASA, Charlson, prostate size, diverticular size, IPSS). The tendency of the diverticulum was to progressively decrease in the first 12 weeks after surgery and during following months (Figures 1-2). The catheter was removed at a median time of 10 (8-12) days and the median length of stay was 5 (4-6) days. None of the patients required a blood transfusion. No major complications (Clavien-Dindo $>2$ ) were seen, while 2 patients (5.1\%) developed postoperative fever managed with antibiotic therapy. The median IPSS change was - $62 \%$ (-74\% to - $46 \%)$.

\section{Discussion}

The genesis of the diverticula in obstructed patients is determined by the high pressures generated into the bladder during the voiding contraction of the detrusor muscle. During this process, the mucosa extrude through the weakest points of the bladder muscular wall leading to the formation of the bladder diverticula.

The need for treatment of secondary bladder diverticula is a debated topic. Large bladder diverticula may lead to post void residual urine, lithiasis, urinary tract infections and ureteral compression. For these reasons, some authors have proposed several open or mini-invasive laparoscopic and robotic surgical approaches (8-12). These approaches showed to be successful both in terms of disappear of the diverticulum and symptoms improvement despite often very high operative times. Obviously, they were associated to endoscopic surgery in order to manage the causing obstruction.

On the contrary, other authors do not see the need to intervene surgically for these benign pathologies, and they place surgical attention only on the underlying cause (13). Agarwal et al. evaluated the results of patients with bladder diverticula and outlet obstruction undergone HoLEP alone (13). They found a significant improve in the post-void residual, peak flow and symptoms at a 15 months follow-up, and only $6 \%$ of patients requiring diverticulectomy. They concluded that most of patient, even with large diverticula, can avoid more invasive surgical approaches whether the outlet obstruction has been relieved. Recently, an in vivo study on rats showed how large bladder diverticula can alter bladder storage and emptying and can lead to dysfunctional voiding (14). Furthermore, bladder diverticula with a diameter $>5$ $\mathrm{cm}$ seems to increase the risk of acute urinary retention in 
benign prostatic hyperplasia (BPH) patients (15). These studies suggest that, in some cases, the surgical management of large bladder diverticula may be necessary.

Several reports in literature have described the use of endoscopic management of bladder diverticula. Orandi et al. published the first report on a series of 17 patients who underwent transurethral fulguration: a complete resolution was achieved in five patients and a decrease in volume in nine (1). Likewise, Clayman et al. managed six patients obtaining a complete disappear of the diverticulum in five of them and a size reduction in the other (3). Yamaguchi et al. reported the most recent and largest series of bladder diverticula fulguration in 1992: twentysix out of thirty-one patients showed a complete resolution (16). In all these studies the size of the managed diverticula was heterogeneous.

Recently, some authors have proposed the endoscopic management with good success also for large diverticula (4-6). The complete disappearance of the diverticulum may take several months and it depends mainly on the initial dimensions of the diverticulum itself. The pathophysiological process behind these results is not completely clear. The effect of the fulguration of the mucosa may involves also the underlying submucosa, causing ischemia of the diverticular wall and leading to a consequent progressive atrophy. This may determine the progressive retraction and the final disappearance of the diverticulum. Data from our study shows that it's effective in terms of symptoms improvement, with low complication rate and a very short operative time. It adds only few minutes to the mandatory management of the causing obstruction (TUIP/TURP/HoLEP/ThuLEP/etc), it's safe, cost-effective and if it fails it doesn't preclude a subsequent further management through a laparoscopic or robotic approach. With the limit of the small samples, it showed similar outcomes whether performed with monopolar or bipolar energy.

The fact that is retrospective and the absence of a group of control undergone another procedure are the main limits of the study. However, we believe it has been successful in showing the possible role of this mini-invasive surgical approach.

\section{Conclusions}

Although the endoscopic management of large bladder diverticula doesn't guarantee the same successful rate and radicality of other open or minimally-invasive procedures it showed some advantages such a very short operative time and the possibility to be performed under spinal anesthesia (8-10). Furthermore, patients in which the procedure fail can still be further managed with a subsequent different and more radical approach. For these reasons we believe it should be considered as a useful option for patients at risk for major or laparoscopic procedures.

\section{REFEREnCES}

1. Orandi A. Transurethral fulguration of bladder diverticulum: new procedure. Urology. 1977; 10:30-32.

2. Vitale PJ, Woodside JR. Management of bladder diverticula by transurethral resection: re-evaluation of an old technique. J Urol. 1979; $122: 744$.
3. Clayman RV, Shahin S, Reddy P, et al. Transurethral treatment of bladder diverticula. Alternative to open diverticulectomy. Urology. 1984; $23: 573$.

4. Pham KN, Jeldress C, Hefty $T$, et al. Endoscopic management of bladder diverticula. Rev Urol. 2016; 18:114-117.

5. Chandhoke RA, Ghoniem GM. Transurethral electrovaporization of bladder diverticulum: an alternative to open or laparoscopic bladder diverticulectomy. J Endourol Case Rep. 2015; 1:11-13.

6. Pacella M, Mantica G, Maffezzini M, et al. Large bladder diverticula: a comparison between laparoscopic excision and endoscopic fulguration. Scand J Urol. 2018; 52:134-138.

7. Dindo D, Demartines N, Clavien PA. Classification of surgical complications: a new proposal with evaluation in a cohort of 6336 patients and results of a survey. Ann Surg. 2004; 240:205-213.

8. Rifaioglu MM, Warman I, Rassweiler J, Gözen AS. Robotic-assisted bladder diverticulectomy. Cent European J Urol. 2016; 69:238.

9. Cacciamani G, De Luyk N, De Marco V, et al. Robotic bladder diverticulectomy: step-by-step extravesical posterior approach technique and outcomes. Scand J Urol. 2018; 52:285-290.

10. Altunrende F, Autorino R, Patel NS, et al. Robotic bladder diverticulectomy: technique and surgical outcomes. Int J Urol. 2011; 18:265-271

11. Tufek I, Mourmouris P, Argun OB, et al. Robot-Assisted Bladder Diverticulectomy with Concurrent Management of Bladder Outlet Obstruction. Urol Int. 2016; 96:432-7.

12. Porpiglia F, Tarabuzzi R, Cossu M, et al. Is laparoscopic bladder diverticulectomy after transurethral resection of the prostate safe and effective? Comparison with open surgery. J Endourol 2004; 18:73-76.

13. Agarwal DK, Krambeck AE. Holmium laser enucleation of the prostate is an effective treatment in patients with concomitant bladder diverticula and outlet obstruction. World J Urol. 2018; 36:87-90.

14. Celebi S, Kuzdan Ö, Özaydin S, et al. The effect of bladder diverticula on bladder function: An experimental study in rabbits. J Pediatr Surg. 2016; 51:1538-42.

15. Iscaife A, Dos Anjos G, Barbosa C Neto, et al. The role of bladder diverticula in the prevalence of acute urinary retention in patients with $B P H$ who are candidates to surgery. Int Braz J Urol. 2018; 44:765-770.

16. Yamaguchi K, Kotake T, Nishikawa Y, et al. Transurethral treatment of bladder diverticulum. Urol Int. 1992; 48:210-212.

\section{Correspondence}

Guglielmo Mantica, MD

guglielmo.mantica@gmail.com

Department of Urology, San Raffaele Turro Hospital, San Raffaele University Via Stamira d'Ancona 20, 20127 Milano (Italy)

Mauro Pacella

mauropacella@virgilio.it

Nicolò Testino

nicolo.testino@live.it

Matteo Valcalda

matteo.valcalda@gmail.com

Rafaela Malinaric

rafaela.malinaric@gmail.com

Carlo Terrone

carlo.terrone@med.uniupo.it

Department of Urology, Policlinico San Martino Hospital,

University of Genova, Genova (Italy) 